\title{
El enfoque de las capacidades como fundamento de una ética contemporánea*
}

\author{
Wilmer Hernando Silva Carreñoa
}

Resumen: parte de la fundamentación actual de la ética deriva de las teorías y de las escuelas clásicas, particularmente occidentales y de base racionalistas, que se han preocupado por el sentido del buen vivir, incluso hasta normalizarlo. En este artículo se da cuenta del sentido en que el enfoque de las capacidades de Nussbaum puede contribuir a ampliar una fundamentación de la ética contemporánea, usando la fenomenología hermenéutica como ruta y base metodológica, dado que facilita y orienta la interpretación y construcción del discurso. Para lo anterior, primero se describe qué integra el enfoque de las capacidades en Nussbaum; segundo, se tematiza cuál es la comprensión de Nussbaum respecto al ser humano; tercero, se muestra cómo el enfoque de las capacidades difiere de las teorías éticas tradicionales, como la ética de la virtud, el utilitarismo y la deontología; y finalmente, se analiza en qué sentido se configura el desarrollo de la capacidad como fundamento de la dignidad. Se concluye que el enfoque de capacidades, además de posibilitar un espacio en el que podamos hacer juicios interculturales sobre los diversos modos de vida, ofrece alternativas de debate, por ejemplo, al considerar la ética frente a la "discapacidad" como uno de los asuntos de especial interés para la bioética.

Palabras clave: enfoque de las capacidades; dignidad; ética; discapacidad; justicia social

Recibido: 21/02/2020

Aceptado: $30 / 11 / 2020$

Disponible en línea: 23/04/2021

* Este artículo es derivado del proyecto de investigación El enfoque de las capacidades como fundamento de una formación ética y moral contemporáneas frente a la sociedad del rendimiento, desarrollado en la Corporación Universitaria Minuto de Dios. En Bogotá, 2019-2020.

a Doctor en Educación de la Universidad Pedagógica Nacional, magíster en Ciencias de la Educación y licenciado en Filosofía de la Universidad de San Buenaventura, Bogotá. Profesor titular de la Corporación Universitaria Minuto de Dios, Bogotá.

Correo electrónico: wilmer.silva@uniminuto.edu.co ORCID: https://orcid.org/0000-0002-3506-7606 
Cómo citar: Silva WH. El enfoque de las capacidades como fundamento de una ética contemporánea. Rev. latinoam. bioet [Internet]. 23 de abril de 2021 [citado 23 de abril de 2021];20(2): 75-89. Disponible en: https://doi.org/10.18359/rlbi.4615

\title{
The approach to capabilities as the foundation of contemporary ethics
}

\begin{abstract}
: part of the current foundation of ethics derives from theories and classical schools, particularly Western and rationalist-based, which have cared about the sense of good living, even until normalizing it. This article considers the meaning in which Nussbaum's approach to capabilities can contribute to broaden a foundation of contemporary ethics, using hermeneutic phenomenology as a methodological route and basis, since it simplifies and guides the interpretation and construction of discourse. For this, it is first described what integrates the capabilities approach in Nussbaum; second, the subject of Nussbaum's understanding of human beings is discussed; third, it is shown how the approach to capabilities differs from traditional ethical theories, such as the ethics of virtue, utilitarianism, and deontology; finally, the meaning in which capacity development is configured as the foundation of dignity is analyzed. It is concluded then, that the skills approach, in addition to enabling a space in which we can make intercultural judgments on the diverse ways of life, offers alternatives of debate, for example, by considering ethics against "disability" as one of the issues of special interest to bioethics.
\end{abstract}

Keywords: approach to capacities; dignity; ethics; disability; social justice

\section{O enfoque das capacidades como fundamento de uma ética contemporânea}

Resumo: parte da fundamentação atual da ética deriva das teorias e das escolas clássicas, particularmente ocidentais e de base racionalistas, que se preocuparam com o sentido do bom viver, inclusive até normalizá-lo. Neste artigo apresenta-se o sentido em que o enfoque das capacidades de Nussbaum pode contribuir para ampliar uma fundamentação da ética contemporânea, usando a fenomenologia hermenêutica como rota e base metodológica, já que facilita e orienta a interpretação e a construção do discurso. Para isso, primeiro descreve-se o que integra o enfoque das capacidades em Nussbaum; segundo, tematiza-se qual é a compreensão de Nussbaum com relação ao ser humano; terceiro, mostra-se como o enfoque das capacidades difere das teorias éticas tradicionais, como a ética da virtude, o utilitarismo e a deontologia; e finalmente, analisa-se em que sentido se configura o desenvolvimento da capacidade como fundamento da dignidade. Conclui-se que o enfoque de capacidades, além de possibilitar um espaço no qual podemos fazer julgamentos interculturais sobre os diversos modos de vida, oferece alternativas de debate, por exemplo, ao considerar a ética diante da "deficiência" como um dos assuntos de especial interesse para a bioética.

Palavras-chave: enfoque das capacidades; dignidade; ética; deficiência; justiça social 


\section{Introducción}

Los problemas de la globalización han desatado una gran controversia desde hace varias décadas. La globalización, ampliamente interpretada, se manifiesta en diversas formas de actividad social, incluida la vida económica, política y cultural, y lo particular de tal fenómeno es, justamente, la ausencia de fronteras. Por lo que preguntar por una ética global implica un razonamiento moral más allá de las fronteras, las cuales pueden involucrar a la cultura, la religión, etnia, género, raza, clase, sexualidad, ubicación geográfica, experiencia histórica, medio ambiente, especies, naciones, etc. Lo anterior ha sido la base de ocupación de muchos especialistas en ética, pues se preguntan cómo abordar mejor los problemas de la globalización, es decir, cómo comenzar a abordar los conflictos que surgen cuando colisionan normas, valores y prácticas culturales muy diferentes.

En esta línea cabe destacar la obra editada por Thunder (1), que recopila varios ensayos académicos en los que se ofrecen debates reflexivos sobre los desafíos claves que enfrenta la ética en un mundo globalizado, caracterizado especialmente por la fragmentación socioeconómica y la multiculturalidad. Aun cuando variadas tradiciones filosóficas subyacen a esta colección, puede indicarse que todos sus ensayos concuerdan en que la crisis de la ciudadanía contemporánea es una crisis de los valores éticos que dan forma y significado a la vida social actual. Temas como el patriotismo frente a una ciudadanía global, la objeción de conciencia en una sociedad moralmente diversa, el problema de la solidaridad ciudadana frente a la injusticia nacional y global y la aceptación universal de principios humanitarios son, entre otros, algunos de los núcleos del debate que esta obra expone.

Ahora, aunque requiere un mayor estudio, puede decirse que existen dos amplios enfoques (por demás filosóficos) para abordar el desacuerdo y el conflicto moral transfronterizo. El enfoque dominante tiene como objetivo desarrollar teorías morales que no están comprometidas con una sola cosmovisión o incluso alguna base religiosa, pero que son compatibles con varias perspectivas. Este enfoque, que puede denominarse universalista, busca desarrollar una teoría que tenga como base un conjunto de normas y principios universales, y que, a la vez, sea amplio, es decir, que pueda tener una concepción sólida del bien dentro de un contexto particular y que respete las tradiciones locales. En este enfoque se pueden ubicar a los teóricos de los derechos humanos, la deontología en general y la ética del discurso, desarrollada particularmente por Benhabib (2).

El otro enfoque, defendido por Walzer (3) principalmente, es el comunitarismo. Tal enfoque niega la posibilidad de desarrollar o establecer una única base universal que brinde principios universales y que, a la vez, pueda respetar el pluralismo y la diversidad cultural. Las principales teorías éticas tradicionales han procurado dar respuesta a este debate, por esto, en este artículo se hace un análisis del fundamento ético en el enfoque de las capacidades y de su relación con tales teorías tradicionales de la ética, para mostrar en qué sentido desde los planteamientos de Martha Nussbaum puede entreverse que el enfoque de las capacidades ofrece una salida a este desencuentro, es decir, puede proporcionar una base universal del desarrollo humano y, a la par, respetar las diferencias religiosas y culturales.

Conviene señalar que en el desarrollo del proyecto de investigación, del que es derivado este artículo, se recurrió a la fenomenología hermenéutica como la base metodológica apropiada, toda vez que esta se ocupa del mundo de la vida o de la experiencia humana tal como se vive, en otras palabras, el punto nuclear de la investigación es la vivencia misma, por lo que el propósito es crear significado y lograr un sentido de comprensión de las vivencias, como sostienen Wilson y Hutchinson (4).

La fenomenología de la práctica o fenomenología hermenéutica deviene como un camino metodológico centrado en la semántica que surge de la interacción interpretativa entre los textos y el lector, es decir, quien lee está en condición de dar sentido. Max van Manen afirma que "hermenéutica significa que la reflexión sobre la vivencia debe regirse por un lenguaje discursivo y por dispositivos interpretativos sensibles que hacen posible e inteligible el análisis, la explicación y la descripción 
propias de la fenomenología" (5, p. 29). En palabras de Manen, la donación de sentido implica que la fenomenología hermenéutica es un método "para preguntar no un método para responder o descubrir o bosquejar conclusiones determinadas" (5, p. 32). Para el caso, se trata de dar sentido a la comprensión de la ética desde el enfoque de las capacidades de Nussbaum, o lo que es configurar temáticamente.

De acuerdo a lo anterior, para el análisis de sentidos temáticos se desarrollaron tres etapas generales: primera, caracterización pre-reflexiva; segunda, configuración temática; y tercera, análisis temático o construcción de las variaciones de sentido. En la primera etapa se trata de que "una vez he permitido la emergencia del fenómeno, (...) puedo valerme de mis vivencias previas para encontrar sobre éste diversas variaciones temáticas, esto es construirlo y reconstruirlo interpretativamente" (6, p.112), en este sentido, se construye y caracteriza de qué trata el enfoque de las capacidades desde Nussbaum. En la segunda, se configura los puntos de comprensión de algunas de las teorías éticas clásicas y en la tercera, se reconstruye las variantes distintivas del enfoque de las capacidades respecto a dichas teorías como fundamento de una ética actual.

\section{Tematización del enfoque de las capacidades}

Vale reseñar que el enfoque de las capacidades, un enfoque de carácter normativo, se desarrolló a partir de la década de 1980 principalmente por el economista Amartya Sen y la filósofa Martha Nussbaum. Sin embargo, sería Nussbaum quien en gran medida desarrollara el enfoque de las capacidades como una base filosófica para indagar y cultivar el desarrollo humano en el mundo.

Se inicia con Amartya Sen, este teórico economista y fundador del enfoque de las capacidades desarrolló su teoría con el fin de identificar un espacio en el que se puedan hacer juicios interculturales sobre la calidad de vida. Para entender mejor cómo se puede aprobar esto, debemos indicar una distinción crítica hecha por los defensores del enfoque de las capacidades, entre la función (o funcionamiento) y la capacidad. Por un lado, según Sen (7), un funcionamiento es un logro, pero debe entenderse ampliamente que incluye cualquier estado del ser. Examínese el ejemplo de Sen de montar en bicicleta que arroja luz sobre un 'funcionamiento' (8, p. 30). Un ciclista es tal porque ha logrado el propósito de lo que uno hace con una bicicleta, es decir, montarla. Puede decirse claramente que la elección de andar en bicicleta es una función de un ser humano, sin embargo, el alcance del funcionamiento no se limita simplemente a la intención de una persona de andar en bicicleta.

Un funcionamiento implica cualquier estado de ser que incluye alegría, felicidad y miedo. Por ejemplo, un niño que comienza a andar en bicicleta puede mostrar una gran cantidad de miedo mientras se tambalea por el camino, pero una vez que entiende cómo andar en bicicleta sin problemas, puede disfrutar (y quizás entusiasmarse) de andar en bicicleta. Por lo tanto, cuando el niño monta su bicicleta (y está entusiasmado de hacerlo) ha realizado el funcionamiento de andar en bicicleta y de tener las emociones asociadas con hacerlo mientras desarrolla a la vez, verbigracia, la capacidad de jugar y de divertirse. Así que el funcionamiento de una capacidad no es aislado, conlleva a otros funcionamientos.

En este sentido, una capacidad es una posibilidad, pero no es cualquier posibilidad, es una real. A saber, se puede hablar sobre la posibilidad de que una persona en un área profundamente golpeada por la pobreza encuentre empleo y mantenga a una familia, sin embargo, tal posibilidad puede no ser real al tener en cuenta las circunstancias externas, sin ropa, comida o refugio.

Dicho de otra manera, en el enfoque de Nussbaum y Sen (9), un conjunto de capacidades es el total de funciones disponibles para que una persona las realice. Al describirlo de esa manera, Sen establece una correlación profunda entre la libertad y la función, es decir, cuanto más limitada es la libertad de una persona, menores son las oportunidades que esta tiene para cumplir sus funciones. En resumen, de acuerdo con Sen (8, p. 102), una capacidad $x$ implica tener la posibilidad real para $\mathrm{x}$, la cual depende de mis poderes, y que no haya circunstancias externas que me impidan realizar $\mathrm{x}$. 
Una capacidad y una función no deben entenderse como mutuamente excluyentes o completamente paralelas entre sí. Consideremos dos personas con las mismas capacidades, a pesar de que tienen las mismas capacidades, pueden participar en funciones radicalmente diferentes. Dos personas pueden tener la oportunidad de participar en el juego, pero lo hacen de maneras radicalmente diferentes (por ejemplo, uno puede nadar o divertirse mientras el otro se ofrece como voluntario en un refugio para personas sin hogar). Los defensores del enfoque de las capacidades argumentan que esto hace que la teoría sea más atractiva, es decir, acomoda varias formas de vida a pesar de que presenta una concepción del bien.

Ahora, consideremos una situación en la que las personas participan en las mismas funciones, pero poseen diferentes capacidades establecidas. Tomemos el hambre, de acuerdo con el ejemplo de Sen (10). Dos personas pueden estar hambrientas, no obstente, esta situación puede darse por razones radicalmente diferentes, por un lado, hay una persona que busca satisfacer su deseo de comer, sin embargo, no puede debido a las circunstancias socioeconómicas; por otro, una persona que está hambrienta porque está ayunando por razones religiosas o protestando por una injusticia. En ambos ejemplos la persona sufre de inanición por razones completamente distintas. En síntesis, para Sen (7) la capacidad es una posibilidad auténtica que tiene la persona para llevar a funcionamiento, aun cuando las razones y motivos para la acción sean diferentes.

Ahora se mencionará la perspectiva asumida por Martha C. Nussbaum. En su enfoque de capacidades, la perspectiva de esta autora tiene deuda con Aristóteles y con J.S. Mill (11). Al igual que Sen, ella aborda la distinción de capacidades y funcionamientos, al hacerlo Nussbaum (12) argumenta que una función no debe realizarse de cualquier manera, sino de una manera verdaderamente humana, es decir, si una persona vive una vida en la que no puede ejercer sus poderes humanos (por ejemplo, creatividad autoexpresiva), entonces, está viviendo su vida de una manera más animal que como ser humano.
Nussbaum busca un enfoque de capacidades que pueda expresar plenamente los poderes humanos, y no solo proporcionar oportunidades (reales) para que las personas realicen ciertas funciones. En otras palabras, ella no niega, como argumenta Sen, que una capacidad es una posibilidad u oportunidad real para que un individuo realice ciertas acciones, no obstante, eso es meramente necesario y no suficiente para el enfoque de capacidades. De acuerdo con Nussbaum (13), a Sen le falta aspectos que son particularmente exclusivos de los seres humanos, esto son los poderes humanos. Nussbaum (14) entiende que la distinción entre capacidades y funciones se realiza de manera múltiple, es decir, mientras que las capacidades son el espacio para la oportunidad de acciones particulares, la forma en que se manifiesta ese espacio, a través de diferentes acciones, es el funcionamiento de una persona.

Esta autora también señala que hay tres diferencias específicas entre su enfoque de las capacidades y el enfoque de Amartya Sen. En primer lugar, Nussbaum (12) acusa a Sen de no rechazar explícitamente el relativismo cultural, ella está de acuerdo con sus simpatías por las normas universales, pero critica su incapacidad para rechazar por completo el relativismo cultural. En segundo lugar, Nussbaum critica a Sen por no basar su teoría en una idea del verdadero funcionamiento humano. Y en tercer lugar, Sen no proporciona una lista explícita de capacidades centrales, de hecho, Sen ha sido crítico con el intento de proporcionar una lista de capacidades centrales.

Nussbaum clasifica las capacidades en tres tipos, básicas, internas y combinadas. Las capacidades básicas son el equipo innato de las personas, el cual es la base necesaria para desarrollar capacidades más avanzadas. Nussbaum señala que la mayoría de los bebés, por ejemplo, tienen las capacidades básicas para la razón práctica y la imaginación, aunque sin desarrollo y educación no pueden usarla. Las capacidades internas son estados o condiciones suficientes de las personas para el ejercicio de una función correspondiente (dado el complemento adecuado de las condiciones externas). Estas se basan en capacidades básicas preexistentes mediante procesos, tales como 
el ejercicio, la educación y la capacitación. La mayoría de los adultos tienen las capacidades internas de uso del habla, las cuales no existirían sin la educación informal que se produce junto con la socialización. Lo anterior indica que muchas capacidades internas requieren un entorno educativo para su desarrollo.

Por su parte, las capacidades combinadas surgen de la relación de las capacidades internas y las condiciones externas que hacen que el ejercicio de una función sea una opción activa. Esto requiere, entonces, de la promoción de capacidades internas (mediante educación o capacitación) y la disponibilidad de las condiciones externas, a saber, institucionales y materiales.

\section{La idea básica sobre el ser humano en Nussbaum}

Martha Nussbaum dice que el pensamiento rector detrás de su enfoque es "uno que yace en el corazón del proyecto de Rawls (...) la idea del ciudadano como un ser humano libre y digno" (16, p.46). Conviene precisar que la concepción política de la justicia de John Rawls (16) implica el compromiso de los ciudadanos de reconocerse mutuamente como personas libres e iguales, dentro del marco de un orden social concebido según el modelo de Estado-nación. Por eso el enfoque de Nussbaum (15) es cosmopolita, o mejor, internacionalista.

Nussbaum sostiene una visión liberal del ser humano. En el centro de esta tradición del pensamiento político liberal hay una doble intuición sobre los seres humanos. La primera es que todos tienen la misma dignidad y valor al ser humanos, sin importar dónde se encuentren en la sociedad, y la segunda es que la fuente principal de este valor es un poder de elección moral dentro de ellos, un poder que consiste en la capacidad de planificar una vida de acuerdo con la propia evaluación de los fines.

A estas dos ideas Nussbaum (15) vincula una más, que se relaciona con "la igualdad moral de las personas les da un justo reclamo a ciertos tipos de tratamiento a manos de la sociedad y la política" $(15$, p. 57). Tal tratamiento que se reclama implica que se respete y se promueva la libertad de elección y el igual valor de las personas que eligen. Nussbaum sostiene que "el núcleo de la personalidad racional y moral es algo que todos los seres humanos comparten, aunque puede ser de diferentes maneras por sus diferentes circunstancias sociales" (17, p.70). Por eso, su punto de vista es que la teoría política debería basarse en "la concepción de los seres humanos como agentes esencialmente racionales" (17, p.71).

En el corazón de la teoría liberal de la justicia y de los derechos humanos de Nussbaum está su versión del concepto de Amartya Sen (7) de libertades o capacidades sustanciales. Sen desarrolló esta noción como una forma de abordar las cuestiones de la justicia y el desarrollo humano.

En Nussbaum, la pregunta por lo justo tiene siempre un correlato. Si se dice, por ejemplo, que la distribución es injusta, se tiene que saber de qué es la distribución. En relación con el desarrollo humano, específicamente sobre la pobreza, la pregunta es saber precisamente qué debe esforzarse por aumentar, por eso, el enfoque de las capacidades se concentra en lo que pueden hacer y ser realmente las personas. No obstante, es importante destacar que el enfoque de las capacidades considera a las personas una por una, no agrupa a los individuos en familias, y no ignora las relaciones y las distribuciones desiguales de poder dentro de las familias. De la misma manera, el enfoque de las capacidades no se ocupa solamente de la distribución de los recursos, dado que estos no tienen ningún valor en sí mismos desconectados de la promoción del funcionamiento humano, es decir, de lo que los seres humanos realmente hacen y son.

\section{El enfoque de las capacidades y las teorías éticas tradicionales}

Las teorías éticas que han dominado la filosofía occidental son principalmente la ética de la virtud, el utilitarismo y la deontología. El enfoque de las capacidades no se puede reducir a ninguna de estas teorías éticas, empero, sí hay que señalar que está en deuda más o menos con cada una de ellas y, especialmente, que tiene puntos precisos de desencuentro con las mismas. 


\section{1 Ética de la virtud}

El enfoque de las capacidades de Nussbaum tiene como punto de partida la comprensión de la virtud en Aristóteles (17). A pesar de que hay claras diferencias entre la tradición de la virtud y la propuesta de Nussbaum, es un idea fundamental, puesto que Nussbaum busca una teoría que sedimente el por qué los seres humanos deben usar sus poderes para florecer de una manera verdaderamente humana.

En términos generales, el enfoque de las capacidades, al igual que la ética de la virtud, afirma que los seres humanos deben ejercer sus capacidades (o potencias) para vivir bien como humanos. Aunque los neoaristotélicos contemporáneos se esfuerzan por dar una explicación del desarrollo humano desde una comprensión naturalista mediante la psicología empírica, en el enfoque de Nussbaum, sin embargo, se comprende la explicación de Aristóteles del funcionamiento como un mero concepto moral no naturalista. De la misma manera, a diferencia de otros neoaristotélicos (y del mismo Aristóteles), Nussbaum (13) no tiene la intención de proporcionar una doctrina integral del desarrollo humano, pues ella cree que basta con indicar, en principio, una lista tentativamente completa de capacidades. Esto último porque solo se trata de un umbral mínimo para garantizar la calidad de vida de las personas. Umbral que, por supuesto, se ampliará conforme a las necesidades y características de los contextos de cada nación o Estado.

Otro punto de distanciamiento entre el enfoque de Nussbaum y la ética de la virtud está dado en la necesidad de la construcción del carácter y la motivación de los actos de las personas. A Nussbaum (14) no le interesa dar cuenta de por qué las personas realizan ciertas acciones o construyen su carácter durante un período a través de motivaciones adecuadas, más bien le preocupa dar cuenta de la importancia de proporcionar el espacio adecuado que le permita a una persona usar sus capacidades para cumplir una función, si así lo desea.

Esto no significa que para Nussbaum la motivación carezca de sentido e importancia, sino que debe verse con un énfasis distinto. Nussbaum (12) argumenta que los deseos informados (es decir, la justificación para el enfoque de las capacidades) no pueden ser ningún anhelo, sino aquellos que contribuyen siempre a vivir bien. Verbigracia, a pesar de que una persona puede desarrollar la capacidad de la razón práctica a través de la educación, no debe usarla de tal manera que coaccione a los demás. Tal deseo sería cuestionado por Nussbaum, ya que, por un lado, impide que la persona forzada participe de todas las capacidades y, por otro, no refleja un deseo informado.

\subsection{El enfoque de las capacidades respecto al comunitarismo}

Por un lado, el comunitarismo puede resumirse en una crítica de la teoría liberal y, por otro, en la importancia de las normas políticas o sociales dentro de una comunidad. A grandes rasgos, los teóricos liberales sostienen que un ego es ahistórico, asocial y apolítico, por lo que no necesariamente depende de las prácticas y de las creencias de su comunidad, por el contrario, puede emanciparse de estas. Sandel (18), un nacionalista-comunitario, explica que un ego liberal no está comprometido, es decir, no está casado con una concepción particular del bien, que por demás no ha elegido, por tanto, primero está la posibilidad de elección que el objeto a elegir. Para Nussbaum (12), el concepto de elección es fundamental, dado que le corresponde a la persona decidir si realiza o no una determinada capacidad en su vida. Desde luego, este proceso, que bien puede enmarcarse como la praxis de la deliberación ética, es una forma de estructurar el pensamiento del ser humano en relación con un problema ético. En ese sentido, puede fortalecerse si se basa en un enfoque colaborativo, puesto que muchas de las elecciones de la persona involucran a otros, por lo que, como sugiere Thames (19), se hace necesario llegar a un consenso, es decir, llegar a una posición con la que todos se sientan lo suficientemente cómodos, aunque aún puedan darse elementos menores de desacuerdo.

Alasdair MacIntyre (20), un comunitarista aristotélico, argumenta en contra de la teoría política liberal en su concepción del ego. Este autor sostiene que un ego está incrustado necesariamente en un conjunto particular de creencias culturales, prácticas y en una historia. MacIntyre, siguiendo a 
Aristóteles, afirma que para que un sujeto viva una buena vida debe ser virtuoso; y una virtud, según MacIntyre (21, p.71), es un rasgo del carácter que permite al ser humano lograr bienes que son inherentes a sus prácticas. Conviene señalar que por práctica se refiere a una actividad humana cooperativa y socialmente establecida, a través de la que los bienes inherentes a dicha actividad se realizan en el curso de tratar de alcanzar esos estándares de excelencia, por lo tanto, vivir una buena vida implica ser virtuoso dentro del contexto de una práctica comunitaria dada.

Téngase en cuenta, además, que para los comunitaristas, la justicia se limita y debe concentrarse en las comunidades, en lugar de focalizarse en los seres humanos en sentido general. Es por esto que hay en el comunitarismo una fuerte crítica y rechazo a la noción de que es posible hacer juicios normativos universales. Aunque en las teorías comunitaristas no está claro cómo sería una comunidad virtuosa exactamente, puede indicarse que tendría una concepción de la vida digna en la que las personas se esfuerzan por alcanzarla y pueden tener las condiciones para lograrlo. Esto es claramente contrario al proyecto liberal, en el que los individuos persiguen cualquier concepción del bien que deseen, siempre y cuando no interfieran ni dañen a otro. Puede decirse que si en el comunitarismo se reclama por la igualdad de condiciones, en el liberalismo se aboga por la igualdad de oportunidades, pero en este último caso no se garantiza que efectivamente puedan disfrutarse por parte de cada individuo.

Dicho esto, conviene señalar que Nussbaum simpatiza con el comunitarismo en la medida en que en este reconoce la importancia de las tradiciones y prácticas locales que dan forma a la vida de las personas. No obstante, son más los puntos en contra del comunitarismo por parte de Nussbaum, finalmente puede afirmarse que el enfoque de las capacidades rechaza de plano el comunitarismo. Precisamente, Nussbaum (12) sugiere que los comunitaristas se equivocan porque no reconocen que existe una concepción del individuo o de la persona que no depende de una tradición particular, sino que cada persona debe ser tratada como un fin, merecedora de respeto, dignidad y honor, y esta condición es la base del umbral mínimo para una calidad de vida aceptable. Por este motivo, para Nussbaum las capacidades se basan en la intuición de que cada persona es merecedora de una vida digna, y esta intuición se mantiene independientemente de la comunidad en la que vive.

\subsection{Entronque con la deontología}

Precisar el pensamiento kantiano en el enfoque de las capacidades requiere de un mayor desarrollo. Por ahora, baste con señalar que al fundamentar el enfoque de las capacidades, Nussbaum se vale del procedimentalismo kantiano para justificar su teoría. Especialmente, retoma la concepción kantiana del valor humano, esta es la noción kantiana de que todos los seres humanos tienen un valor intrínseco y, en consecuencia, siempre deben ser tratados como un fin y no como un medio.

Este valor intrínseco integra en Nussbaum su comprensión de la dignidad, entendida como noción general para fundamentar la lista de capacidades, así como una base conceptual que fundamenta los derechos de los animales (especialmente los animales humanos) y como un argumento para justificar la importancia de cultivar el funcionamiento de las capacidades. Recuérdese que la idea central del enfoque de las capacidades de Nussbaum (13) es que todos los seres humanos deben reconocer y respetar el derecho de los demás a vivir y llevar una vida acorde con la dignidad humana.

Esta idea no se restringe únicamente a la condición de la persona que puede valerse racionalmente por sí misma. En efecto, Nussbaum utiliza la noción de dignidad para destacar los derechos y las capacidades de aquellos humanos "con discapacidades" y, también, de los animales no humanos.

Sin embargo, esta deuda de Nussbaum con Kant (21) al fundamentar la dignidad humana termina siendo una fuerte crítica, especialmente en tres aspectos. Primero, el liberalismo kantiano aboga por un sujeto individualista, cuya dignidad poco tiene que ver con el valor de hacer parte de una comunidad o de entidades sociales. Respecto al enfoque de las capacidades, la dignidad tiene sentido en y por la vida comunitaria, hasta el punto de que la filiación es una capacidad y, por ello, 
parte del umbral mínimo que debe garantizarse. El enfoque de las capacidades, por demás, es en sí mismo una fuerte crítica a cualquier ideología que promueve una especie de solipsismo vital. Segundo, la idea kantiana de que todos los seres humanos tienen un valor intrínseco de igualdad se presenta de forma abstracta y formal, en estrecha relación con la necesidad de fundar un principio categórico universal, por lo que poco o nada importan las condiciones y situaciones de vida concretas en las que devienen las capacidades de una persona. Y tercero, toda fundamentación kantiana tiende a centrarse en la razón y su despliegue, dejando de lado otros aspectos de la vida humana importantes como las emociones y la vida afectiva y política.

Con todo, la dignidad humana no se fundamenta en la comprensión metafísica y racional del imperativo categórico kantiano, porque su valor tiene que ver con la posibilidad real de ejecución de las capacidades de la persona. Esta última implica que toda persona no puede sustraerse a los demás, lo anterior se refiere a que el tema de la dignidad y del desarrollo humano no se fundan en el cultivo de proyectos normativos de autosuficiencia o de independencia, sino en la posibilidad de la vida intersubjetiva.

\subsection{Diferencias con el utilitarismo}

El utilitarismo, también denominado consecuencialismo, es una ética normativa proveniente de los filósofos y economistas ingleses de finales del siglo xVIII y xix, Jeremy Bentham (22) y John Stuart Mill (11). El principio base de esta corriente reza que una acción es correcta siempre que promueva y alcance el fin de la acción, es decir, que efectivamente sea útil. Por tanto, esta doctrina ética sostiene que la virtud se basa en la utilidad, y que la conducta debe estar dirigida a promover la mayor felicidad para el mayor número de personas, luego, el fin de toda acción humana ha de ser producir las mejores consecuencias posibles.

En principio, puede confundirse el enfoque de las capacidades con el enfoque consecuencialista, pues Nussbaum (14) está preocupada, por ejemplo, por reducir la pobreza de las naciones en el mundo o por superar la discriminación cultural, estos son fines que afectan a la humanidad en general, y por ello, también son acciones válidas dentro del consecuencialismo.

No obstante, el enfoque de Nussbaum (15) se diferencia del utilitarismo por tres razones, principalmente, a saber: a) mientras que el sujeto del utilitarismo es abstracto, pues está interesado en maximizar la utilidad de todos, el enfoque de las capacidades se interesa por la persona en particular y se pregunta por las necesidades y el contexto propio de cada quien; b) mientras que en el utilitarismo no se les da cabida a las diferencias interculturales (por ejemplo el hecho de que todas las personas llevan vidas muy distintas y en diferentes circunstancias, pues lo que interesa es la utilidad general y no las personas o grupos de personas en particular), en el enfoque de las capacidades no se puede ignorar el bien particular de una cultura, es decir, es necesario identificar, verbigracia, qué bienes deben cultivarse y distribuirse adecuadamente en una región en particular; y c) en el utilitarismo todo bien se valora por su utilidad, esta se mide por el impacto que una acción tiene fuera del sujeto, en otras palabras, para el utilitarismo no son relevantes los aspectos internos del sujeto, como las emociones, pues no se pregunta por cómo se siente el sujeto a quien le sucede algo, y menos se pregunta sobre qué es capaz de hacer o de ser una persona, lo cual, como se sabe, es el núcleo de la capacidad.

De este modo, el utilitarismo, a saber, es un enfoque influyente de preferencia en la administración política y social de los gobiernos de hoy. Este enfoque sugiere que la planificación social debería tratar de maximizar la satisfacción de las preferencias que los individuos tienen antes de que se les apliquen las políticas sociales (como si las preferencias no se vieran afectadas por los acuerdos sociales). Contrario a esto, el enfoque de las capacidades rechaza el estándar de preferencia como un estándar de lo que es socialmente valioso. Incluso, Nussbaum (14) señala que las preferencias pueden distorsionarse o modificarse (por ejemplo, una mujer maltratada, víctima de la violencia doméstica, puede llegar a convencerse de que un maltrato moderado es una situación cómoda y no "preferir" una mayor libertad). 


\section{La capacidad como base de la vida digna}

Ahora, se tendrá en cuenta otro asunto de especial relevancia. Así como deviene el yo puedo, el homo capax (23), el sujeto de capacidades; también es factible la emergencia del yo no puedo, del no soy capaz. Andersons (24) toma el ejemplo de un individuo que se preocupa por jugar bien a las cartas. Sin duda, un buen jugador de cartas es indicador de un buen funcionamiento de capacidad, además, se podría inferir que algunas personas pueden valorar razonablemente el funcionamiento de las capacidades en su concepción de una buena vida.

Para empezar, no hay nada malo con las personas que buscan ser buenas jugando a las cartas, sin embargo, como dice Anderson, ser un pobre jugador de cartas no hace que uno sea débil. Más precisamente, el orden social puede y debe organizarse de modo que la habilidad de una persona en las cartas no determine su estado en la sociedad civil, y menos aún su dignidad. Tampoco es necesario ser un buen jugador de cartas para ser un ciudadano. Simons (25) señala que el horizonte y el valor fundamental de la ética se constituyen en la configuración de una disposición para la vida comunitaria, especialmente, para construir una manera participativa de buen vivir. Esto implica una forma de pensar, actuar e intervenir en y para la vida colectiva, además, implica el respeto hacia los otros seres humanos, la defensa de la vida, de la naturaleza y de la realización comunitaria. En ningún sentido, el alcance de lo anterior no está condicionado, y no puede estarlo, a la adquisición de habilidades particulares.

En el centro está, pues, la pregunta por la dignidad. Y esto viene a caso por la llamada capacidad disminuida, que es especialmente entendida desde la psicología. La capacidad disminuida es una condición en la que ya no se es capaz de tomar decisiones efectivas sobre la vida. También se le llama pérdida o falta de competencia propiamente de autonomía. La capacidad puede referirse a la acción de poder decidir cosas (capacidad de decisión) o a la acción para tomar medidas efectivas (capacidad de ejecución).
¿Por qué sucede esto? (lo anterior se refiere a que una persona siendo capaz de hacer algo, ya no pueda serlo). Los estudios científicos sugieren que la capacidad disminuida se produce como resultado de un daño al cerebro. Por ejemplo, la demencia (una disminución en su memoria o en sus habilidades de pensamiento) puede producir una condición en la que ya no recuerda cómo se supone que debe comportarse. Las condiciones psicológicas, como la esquizofrenia (procesos de pensamiento fragmentados combinados con delirios e ilusiones), pueden crear una situación en la que ya no se percibe la realidad de manera efectiva, o la psicopatía, que es un estado en el que se tiene una capacidad limitada para experimentar emociones, y en la que puede que no se entienda las consecuencias o implicaciones morales de sus decisiones y acciones. Así mismo, el daño a los centros de procesamiento emocional, como por un golpe físico en la cabeza, puede hacer que la persona no pueda controlar sus emociones.

En cada uno de estos casos puede considerarse que se tiene una capacidad disminuida, lo que generalmente significa que la sociedad no le permitirá permanecer completamente independiente. Empero, esto merece un estudio un poco más reflexivo, propiamente ético. No se puede decir que una persona florece, de acuerdo con el enfoque de capacidades, si ella no puede realizar las funciones que participan en las capacidades. Esto plantea preguntas interesantes sobre las personas con "discapacidades", en la medida en que pueden tener impedimentos físicos o mentales para realizar ciertas funciones. En consecuencia, se configura uno de los grandes problemas éticos actuales, o lo que es un asunto de estudio de la bioética. En efecto, como lo aborda Carvajal (26), la bioética puede tener sustento sólido hoy en la teoría del desarrollo humano y de las capacidades, expuesta ampliamente por Nussbaum, esto se da, especialmente, porque los problemas que emergen actualmente en el estudio de la ética involucran la disposición de la bios (por lo que en gran parte los problemas éticos actuales serían del ámbito de la bioética) y la ciudadanía. Incluso si se trata de la discapacidad cognitiva, como lo trabaja Canto (27). 
En su formulación temprana de la lista de capacidades, Nussbaum excluyó a muchas personas de la capacidad de vivir una vida verdaderamente humana, ya que ella requería que una vida como esa incluyera el uso de los cinco sentidos, por ejemplo. No obstante, posteriormente se retractó. Nussbaum (28) señala que sería difícil imaginar a una persona viviendo una vida verdaderamente humana con una falta total de sentidos, imaginación y razonamiento.

De hecho, Nussbaum ha ampliado su descripción del funcionamiento de una manera verdaderamente humana (es decir, para la dignidad humana). Ella cree que los "discapacitados mentales", por ejemplo, pueden ganar dignidad no solo desde la racionalidad, sino también a través del apoyo y mediación de las "capacidades de la vida, la salud y la integridad corporal, la estimulación de los sentidos, la imaginación y el pensamiento" $(13$, p.113). Lo anterior indica que no hay nunca una persona discapacitada, sino que esta requerirá de la mediación y ayuda para su despliegue, por ello se sugiere hablar de capacidad asistida. En este sentido, sí hay una clara responsabilidad del Estado para que no solo permita que ocurra tal estimulación de los sentidos, sino que realmente se proporcionen los recursos para que se produzca dicha estimulación.

Ahora, hay preguntas sobre cómo implementar políticas que brinden la mejor oportunidad para que las personas con discapacidad o con capacidad asistida realicen funciones que cumplan con las capacidades. La reflexión ética desde el enfoque de las capacidades tiene mucho que aportar en esto. De una parte, puede sentar las bases para permitir la emergencia en el mundo actual de la persona como un sujeto de capacidades, capaz de actuar, de decidir y capaz de construir historia. De otra, lejos de promover sistemas universales de juicio y valor, puede atender y promover la vuelta a la individualidad humana, es decir, en lugar de agrupar a todas las personas discapacitadas en un solo grupo abstracto, puede volver la mirada a caso por caso, lo que podría, en efecto, implicar a varias personas. Se recuerda que este enfoque permite que cada quien reciba la atención adecuada que necesita.
Desde el enfoque de las capacidades de Nussbaum, el concepto de justicia sería el punto de partida para atender la problemática de la vulnerabilidad social, especialmente, de las personas con diversidad funcional, conforme lo aborda Aparicio (29). Garantizar el funcionamiento de sus capacidades básicas es, de entrada, el reto principal de los retos bioéticos que exige una visión ético-política de accesibilidad estatal y social en el mundo contemporáneo, que, como lo aborda Byung Chul-Han (30), se ve reducido y condicionado al paradigma al rendimiento, aun cuando esto es un asunto que requiere un mayor estudio.

\subsection{La teoría de la justicia social como fundamento de la dignidad humana}

Dado que emerge con esto el tema de la dignidad en el campo de la bioética, conviene recordar que Richard Ashcroft (31) sugirió que la capacidad (y el funcionamiento de esta), en particular desde la perspectiva de Nussbaum, constituye una plataforma importante de sedimentación, lo anterior dentro de su reseña de las bases contemporáneas para una fundamentación bioética de la dignidad (p.679).

Como argumenta García (32), para Nussbaum ni la racionalidad ni las capacidades en sí mismas sostienen la dignidad humana, sino que esta descansa en lo que ella denomina un "umbral mínimo de justicia y dignidad", integrado inicialmente por una lista de diez capacidades humanas, no obstante, está susceptible de ampliarse (23). El interés por asegurar el principio de la dignidad en las personas pasa, entonces, por el funcionamiento de este umbral mínimo de la dignidad en la vida de las ellas, por ello, puede inferirse que la dignidad reclama el funcionamiento de las capacidades. En otras palabras, la dignidad humana deriva de la posibilidad del funcionamiento de la capacidad de los seres humanos para la autonomía. No obstante, conviene precisar que la dignidad no puede fundarse en la presunción o simple declaración del umbral de capacidades en las personas (como un agente racional dotado de libertad y autonomía), sino que se requiere del funcionamiento o despliegue de este 
como sujeto de capacidades. Tal despliegue, como se ha indicado, puede darse a través de la mediación de otros.

¿Cómo asegurar tal funcionamiento? La búsqueda de la dignidad legitima para Nussbaum (15) es la obligación política de asegurar la justica social, y eso es, ante todo, responsabilidad directa del Estado. De ahí que la cuestión de la dignidad es en principio un asunto mismo de la justicia social. En la perspectiva de Nussbaum, es responsabilidad del Estado garantizarla y promoverla.

Por eso, según Nussbaum, la tarea de un Estado solo se cumple con éxito cuando contribuye a que sus ciudadanos lleven una buena vida, esto es, disfruten de su dignidad como personas. Por tanto, la máxima prioridad de las instituciones políticas debería ser la de garantizar que todos los miembros de la sociedad tengan acceso al desarrollo de su dignidad, aunque Nussbaum deja claro que es necesario desarrollar una concepción del bien antes de que se pueda asignar al Estado la tarea de promover una vida buena y justa.

Se tratará ahora de precisar este asunto. Nussbaum explica que el dinero es solo un medio para un fin y no un fin en sí mismo. Entonces, si una distribución uniforme del dinero tiene diferentes efectos en diferentes personas y les permite hacer varias cosas, una distribución tan uniforme no es justa. No es la cantidad de bienes o recursos lo que es decisivo, según Nussbaum, sino lo que se puede hacer con ellos. En este contexto, como señala Nussbaum (15), solo cuando se da cuenta de lo que están haciendo y lo que pueden hacer con sus vidas las personas, qué actividades importantes son promovidas o bloqueadas por ellos y cómo los diferentes conceptos de distribución afectan estas actividades es que un Estado o en una comunidad puede saber cuánto debe darse a quién y bajo qué condiciones institucionales. Para ilustrar esto, Nussbaum explica que, por ejemplo, una mujer embarazada necesita más que una mujer no embarazada, los niños también necesitan menos que los adultos, etc., $y$, en consecuencia, las personas cuyas capacidades solo pueden funcionar de forma mediada necesitarán más que otras.

La consecuencia lógica del argumento de Nussbaum es que se requiere, entonces, de una concepción firme de la dignidad y del bien. Así, antes de que se puedan establecer patrones para distribuir recursos que de alguna manera permitan que cada persona pueda llevar una buena vida, debe definirse con precisión qué es una vida digna, qué actividades humanas son importantes o qué es necesario para una vida humana digna. Esto requiere que el Estado debe centrarse, de un lado, en facilitar que todos los integrantes, sin excepción, puedan disfrutar de una buena vida y, de otro, hacer posible esta buena vida con una distribución equitativa y justa de bienes y recursos. Empero, esto no abroga ni el derecho ni la obligación de cultivar el autodesarrollo humano, que también es fundamental. Las posibilidades que deben ofrecerse a las personas para que puedan llevar una vida digna deben extenderse a todos los ámbitos de la vida. Estas deben tener la oportunidad de funcionar como sujetos de capacidades.

La mera provisión de los recursos necesarios o básicos por sí sola no es conveniente, por lo que además de la capacidad de obtener y utilizar estos bienes, se debe desarrollar e implementar una idea general y vinculante de una vida digna. De hecho, la lista de capacidades que sugiere Nussbaum incluye, entre otras cosas, un sistema de salud uniforme, el suministro de aire y agua saludables, la garantía de seguridad en la vida, el derecho a un alojamiento adecuado, entre otras. Además, asume en su enfoque que no todo el mundo tiene una idea clara de lo que implica vida digna, por eso hace dicha lista. Tener una idea de lo que es una vida digna no se restringe, tampoco, a los intereses de las personas o las culturas, sino que debe construirse de modo colectivo, por eso la tarea del Estado en este sentido.

Nussbaum (15) ejemplifica que en la India las mujeres en algunas regiones comen menos que los hombres, razón por la que están más débiles y se enferman con más frecuencia. Según la opinión, allí los hombres necesitaban más fuerza para trabajar, por lo que merecerían mayor comida, pero esto lo que hace es justificar la desigual distribución. Así que promover una distribución justa, si bien no puede traducirse en una distribución uniforme, tampoco puede darse poniendo en riesgo la calidad de vida de los otros. Cabe señalar que frente 
a este caso en particular Nussbaum argumenta a la inversa, pues dice que las mujeres solo son más débiles y pueden trabajar menos porque menos comida reciben, es decir, porque la distribución de alimentos es injusta (15, p. 122). Las mujeres podrían trabajar igual de bien o mejor si tuvieran la misma cantidad de comida que los hombres, pero como no se tiene una construcción consensuada y justa de lo que es la vida digna o la calidad de vida frente a este caso, señala Nussbaum, las mujeres avalan la distribución desigual de los alimentos.

De esta manera, la teoría de la justicia social en el enfoque de Nussbaum precisa la necesidad de cultivar en las comunidades y en las culturas la idea de lo que constituye una vida digna. Esta es una de las tareas del Estado, es decir, debe ser ejecutada y monitoreada por el Estado, porque solo entonces es posible que cada persona lleve una vida humana digna. El factor decisivo de lo que implica una vida humana digna es que debe ser factible para todos. Las fullerías frente a este propósito son evidentes de inmediato. Una de ellas es que es el estatus social y un conjunto de normas dadas determina lo que alguien imagina como una vida digna, $y$ en consecuencia, lo que considera justo. Pero esto abre otra discusión.

\section{Conclusiones}

Nussbaum muestra que con Rawls existen tensiones inherentes a la teoría que surgen de la conexión entre la doctrina del contrato social y una concepción kantiana de la persona y la reciprocidad de las relaciones de cooperación que entablan las partes contratantes. Con Rawls, los individuos, que establecen las estructuras normativas básicas de su convivencia (en el hipotético estado original detrás de un "velo de ignorancia"), son siempre ciudadanos sensibles, autodeterminados, miembros libres e iguales de la sociedad y libres de cooperar en su vida. Rawls ignora la experiencia humana de severa necesidad física o mental, dependencia temporal o permanente, además, trasladó la inclusión de ciudadanos con discapacidades atípicas al nivel de legislación posterior. Para Nussbaum esto es inaceptable, solo si las partes no saben en su estado original qué "discapacidades" físicas tienen o no podrían tener, establecerán principios que sean realmente justos para las personas con esas discapacidades.

Si bien Nussbaum funda el enfoque de las capacidades con aportes de la noción aristotélica y kantiana de la dignidad humana, advierte que el hecho de poseer la razón como cualidad particular hace que todos los seres humanos sean iguales, con lo que se promueve el universalismo, no obstante, este no se puede construir en detrimento y al margen de la particularidad de las personas y de las culturas. No se cuestiona que la racionalidad y la moralidad están estrechamente conectadas, y que la dignidad humana las implica mutuamente, pues se parte de la base de que la persona y su dignidad son un fin en sí mismo, y no un instrumento para los fines de los demás (33). Empero, esta filósofa sí cuestiona que lo racional se establezca como el único motivo o referente para atribuir o nominar la dignidad de las personas, especialmente con capacidad disminuida o asistida.

El enfoque de las capacidades a menudo se discute en términos de brindar oportunidades (Sen) y poner en funcionamiento capacidades humanas (Nussbaum). La mayoría de las veces es un argumento para reducir la pobreza o aumentar el bienestar de las personas en todo el mundo. Recientemente, ha proporcionado el marco para adelantar argumentos en otras áreas de la ética aplicada, incluida la ética empresarial, el medio ambiente, la ética de las discapacidades y la ética animal. Asuntos que son objeto de estudio por parte del universalismo y del comunitarismo como enfoques éticos dominantes.

Y desde luego el debate entre estos dos enfoques ha sido amplio y no ha tenido, ni eso se espera, un punto final. De una parte, los comunitaristas sostienen y defienden que las normas morales son siempre locales y válidas para una comunidad y un contexto particulares, razón por la que son acusados por los universalistas de promover el relativismo y de no proporcionar métodos ni principios útiles para abordar el conflicto moral transfronterizo. De otra parte, los universalistas son cuestionados por plantear teorías amplias pero muy débiles para ser útiles a cualquier grupo humano, o de fundar sus teorías en premisas que 
no se comparten universalmente $y$, en consecuencia, defender el imperialismo cultural, político y económico.

El enfoque de las capacidades emerge como una salida, toda vez que sedimenta una comprensión de la dignidad humana que atiende a las particularidades y necesidades de las personas y las culturas, a la vez que procura establecer las bases para pensar en la totalidad sin caer en una acción imperialista. A este respecto, conviene citar la obra de Gracia (34) y el trabajo de Pérez y González (35), en los que se destacan los principales desafíos políticos que tiene la educación ética como base clave y necesaria para el mantenimiento de una sociedad sostenible que no ceda a la "moralidad" del mercado, sino que atienda al sentido de lo humano. Debido a que las personas con discapacidades (especialmente graves) están excluidas del establecimiento de principios políticos básicos en las estructuras de las teorías contractuales actuales, sus condiciones de dignidad humana y ciudadanía siguen siendo precarias y su derecho a la igualdad integral está en riesgo.

A nivel teórico, esto complica considerablemente las cosas, puesto que detrás del desconocimiento de lo que las personas son y pueden hacer, las partes ya no pueden simplemente orientarse hacia los más desfavorecidos, porque las posiciones mejores y peores en la sociedad ya no pueden determinarse sobre la base de acceso bienes básicos y recursos. Cualquiera que dependa de una silla de ruedas, por ejemplo, con los mismos ingresos y activos, puede estar significativamente peor que alguien que puede moverse "normalmente", y no necesita ninguna ayuda para compensar su movilidad restringida o poner en funcionamiento su capacidad mediada.

\section{Referencias bibliográficas}

(1) Thunder D. The ethics of citizenship in the 21st century. Switzerland: Springer; 2017. Disponible en: DoI: https://doi.org/10.1007/978-3-319-50415-5

(2) Benhabib S. Cultural complexity, moral interdependence, and the global dialogical community. En: Nussbaum M, Glover J, editores. Women, culture and development. 2a ed. Oxford: Clarendon Press; 2003. p. 11-21. Disponible en: DoI: $10.1093 / 0198289642.001 .0001$
(3) Walzer M. The communitarian critique of liberalism. Political Theory. 1990 febr 1;18(1):6-23. Disponible en: DoI: https://doi.org/10.1177/0090591790018001002

(4) Wilson H, Hutchinson S. Triangulation of qualitative methods: Heideggerian hermeneutics and grounded theory. Qualitative Health Research. [Internet]. 1991 may 1; 1(2):263-276. Disponible en: Dor: https:// doi.org/10.1177/104973239100100206

(5) Manen M. Investigación educativa y experiencia vivida. Barcelona: Idea Books; 2003. 219 p.

(6) Silva W. La fenomenología hermenéutica como método de investigación. En: Silva W, Torres V, Sánchez M, Cano W. y Mendieta M. Desinstitucionalizar la escuela: un análisis crítico desde el desarrollo humano. Bogotá: Instituto Nacional de Investigación e Innovación Social; 2018. 125 p.

(7) Sen A. Development as freedom. Oxford: Oxford University Press; 2001. 370 p.

(8) Sen A. Choice, welfare and measurement. Cambridge: Harvard University Press; 1997. 480 p.

(9) Nussbaum M, Sen A, compiladores. La calidad de vida. México: Fondo de Cultura Económica; 2002. $588 \mathrm{p}$.

(10) Sen A. Resources, values and development. Cambridge: Harvard University Press; 1997. 547 p.

(11) Mill J. S. El utilitarismo. Barcelona: Editorial Alianza; 2007. 144 p.

(12) Nussbaum M. Las mujeres y el desarrollo humano. Barcelona: Herder; 2002. 416 p.

(13) Nussbaum M. Las fronteras de la justicia: consideraciones sobre la exclusión. Barcelona: Paidós; 2006. $425 \mathrm{p}$.

(14) Nussbaum M. Crear capacidades: propuesta para el desarrollo humano. Barcelona: Paidós; 2012. 266 p.

(15) Nussbaum M. Sex and social justice. Oxford: Oxford University Press; 2000. 488 p.

(16) Rawls J. A theory of justice. Cambridge: Harvard University Press; 1999. 539 p.

(17) Aristóteles, Calvo J L, traductor. Ética a Nicómaco. México: Alianza Editorial; 2014. 314 p.

(18) Sandel M. Liberalism and the limits of justice. 2a ed. Cambridge: Cambridge University Press; 2012. 235 p.

(19) Thames B. How should one live? An introduction to ethics and moral reasoning. San Diego: Bridgepoint Education; 2018. 208 p. Disponible en: https://philpapers.org/rec/THAHSO

(20) MacIntyre A. Tras la virtud. Barcelona: Editorial Crítica; 2007. 417 p. 
(21) Kant I. Crítica a la razón práctica. Madrid: Mestas Ediciones; 2004. 220 p.

(22) Bentham J. Deontología o ciencia de la moral (tomo I). Revisada y ordenada por M. J. Bowring. Valladolid: Junta de Castilla y León; 2012. 260 p. Disponible en: http://hdl.handle.net/10481/24324

(23) Silva W. Homo capax: hacia una filosofía de la educación. Bogotá: Editorial Aula de Humanidades y Universidad de San Buenaventura; 2016. 297 p.

(24) Anderson E. Justifying the capability approach to justice. En: Brighouse H, Robeyns I, editores. Measuring Justice. Primary Goods and Capabilities. Cambridge: Cambridge University Press; 2010. p. 81-100. Disponible en: DoI: https://doi.org/10.1017/ CBO9780511810916

(25) Simons A. Ética y ejercio de la ciudadanía. Lima: PUCP; 2017. 140 p.

(26) Carvajal H. Educación en bioética y la formación para la ciudadanía. Praxis \& Saber [Internet]. 2016 en. 4; 7(13):223-42. Disponible en: Dor: https://doi. org/10.19053/22160159.4173

(27) Canto E. Estatus personal en la discapacidad intelectual: considerando las capacidades cognitivas. Revista Latinoamericana de Bioética [Internet]. 2018 jun. 8; 18(35-2):226-241. Disponible en: DoI: https://doi. org/10.18359/rlbi.3137

(28) Nussbaum M. Human capabilities, female human beings. En: Nussbaum M. and Glover J, editors. Women, culture, and development: a study of human capabilities. Oxford: Clarendon Press; 2003; p. 34-71. Disponible en: DoI 10.1093/0198289642.001.0001
(29) Aparicio M. Aproximación bioética a la accesibilidad universal desde el enfoque de las capacidades de Nussbaum. Bioderecho [Internet]. 2017 jul. 20; 5(11):1-11. Disponible en: https://revistas.um.es/bioderecho/article/view/290191

(30) Han B. La sociedad del cansancio. Barcelona: Herder; 2017. 118 p.

(31) Ashcroft R. Making sense of dignity. Journal Medical Ethics [Internet]. 2005 nov. 3; 31:679-682. Disponible en: DoI: https://doi.org/10.1136/jme.2004.011130

(32) García R. La dignidad y sus menciones en la declaración. En: Casado M, Coordinadora. Sobre la dignidad y los principios: análisis de la Declaración Universal sobre Bioética y Derechos Humanos de la UNESCO. Barcelona: Civitas; 2009. p. 43-63.

(33) Nussbaum M. Human dignity and political entitlements. En: Pellegrino E, Schulman A, Merrill T, editores. Human dignity and bioethics. Essays commissioned by the president's Council on bioethics. Washington; 2008. p. 351-80. Disponible en: http:// hdl.handle.net/10822/559351

(34) Gracia J. El desafío ético de la educación. Madrid: Dykinson; 2018. 268 p.

(35) Pérez J. y González P. Los servicios profesionales para las personas con discapacidad. Principios, valores y dilemas éticos. Revista Española de Discapacidad [Internet]. 2018 jun. 8; 6(1):113-37. Disponible en: DoI: https://doi.org/10.5569/2340-5104.06.01.06 
